\title{
ESTILOS DE VIDA DE ESTUDIANTES UNIVERSITARIOS DE CIENCIAS DE LA SALUD
}

\author{
STYLES OF LIFE OF UNIVERSITY STUDENTS OF HEALTH SCIENCES
}

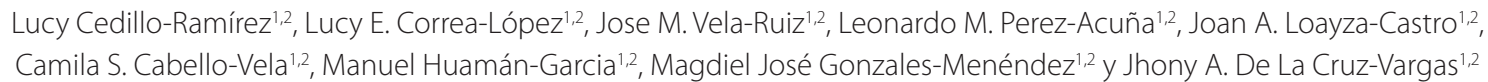

\begin{abstract}
RESUMEN
Objetivos: El objetivo de este artículo es realizar una revisión de las publicaciones que revelen el impacto de los estilos de vida en la salud integral de estudiantes universitarios del área ciencias de la salud. Métodos : La revisión fue efectuada mediante búsqueda electrónica de artículos relacionados con estilos de vida en estudiantes universitarios pertenecientes a las escuelas de ciencias de la salud. Las fuentes de búsqueda fueron ISI, SCIELO, MEDLINE, BVS y PUBMED. Los términos de búsqueda fueron: estilo de vida, estudiantes universitarios y ciencias de la salud. Para esta revisión se seleccionaron los artículos publicados que tuvieron experiencias investigativas desde el 2010 a la actualidad y los que incluyeron aspectos teórico-conceptuales se consideró los publicados desde el año 2000. Resultados: De los 83 artículos revisados, se descartaron 38 por no cumplir con el criterio de haber realizado la investigación en el área de ciencias de la salud, y 12 fueron retirados por ser publicados antes del 2010, quedando solo 33 artículos para esta revisión bibliográfica. De los 33 artículos seleccionados, 18 estudios cumplieron con los criterios de investigación, 15 fueron considerados para incluir aspectos teórico - conceptuales. Respecto a la actividad física, un estudio evidenció que el 40\% de los jóvenes universitarios no realiza ningún ejercicio físico; otra investigación demostró que solo el $44 \%$ de los estudiantes de ciencias de la salud practicaban los recomendados 30 minutos de ejercicio 5 días por semana. Además los patrones alimentarios de los estudiantes fueron inadecuados, haciéndose necesario un cambio en el $90 \%$ de la población, siendo la "comida chatarra" la que más consumieron los participantes. Por otro lado, los datos de un estudio revelaron que 1 de cada 5 de los estudiantes universitarios presentan alteraciones en su salud mental y el consumo de tabaco y/o drogas, aparece como factor de riesgo. Conclusión: La mayoría de los estudiantes universitarios de ciencias de la salud no aplican sus conocimientos en sus propios estilos de vida (alimentación, actividad física y demás prácticas). Esto revela la urgencia de crear programas universitarios que modifiquen y promuevan estilos de vida saludable.
\end{abstract}

Palabras clave: Estilo de vida; Estudiantes universitarios; Ciencias de la salud; Sedentarismo; Actividad física; Alimentación saludable; Tabaquismo; Alcoholismo y bienestar mental (fuente: DeCS BIREME).

\begin{abstract}
Objective: The purpose of this article is to conduct a systematic review of scientific papers that show the impact of lifestyles in integral health of university students who have chosen health sciences careers. Methods: The review was conducted by electronic search of articles related to lifestyles linked to university students from schools of health science. The search sources were ISI, SCIELO, MEDLINE, PUBMED and BVS. The search terms were: lifestyle, university students and health sciences. For this review published articles that had research experiences from 2010 up to date. Those articles which included theoretical and conceptual aspects were considered from 2000 on. Results: 83 articles were reviewed, and 38 were discarded for failing to meet the criteria of having carried out research in the area of health sciences, and 12 were removed because they were published before 2010 . Only 33 articles remained for this bibliographic review. Of the 33 articles selected, 19 studies met the research criteria, 14 were considered because they included theoretical - conceptual aspects. Regarding physical activity, a study showed that $40 \%$ of young university does not perform any physical exercise; other research showed that $44 \%$ of students in health sciences practiced the recommended 30 minutes of exercise five days a week. In addition, the eating patterns of students are inadequate, making it necessary to change it in $90 \%$ of the population, being the "junk food" which is eaten much more by the participants. On the other hand, data from a study revealed that 1 in 5 university students have alterations in their mental health and smoking and / or consumption of drugs appears as a risk factor. Conclusion: Most university students of health sciences do not apply their knowledge on diet, physical activity and other practices that defined their lifestyle. This reveals the urgency of creating university programs that modify and promote healthy lifestyles.
\end{abstract}

Key words: Lifestyle; University students; Health sciences; Physical activity; Healthy eating; Sedentary lifestyle; Smoking; Alcoholism and mental well-being. (source: MeSH NLM).

'Instituto de investigación de ciencias biomédicas (INICIB), Grupo ESVIBUR.

2 Facultad de Medicina Humana, Universidad Ricardo Palma.

Correspondencia: INICIB, Facultad de Medicina Humana, Edificio I. 2 do piso. Campus Universitario. Dirección: Av. Benavides 5430, Surco. Lima, Perú. C.P. 04360. Teléfono: 56232300 Ext. 43034. Correo : lucy.correa@icloud.com

Citar como: Lucy Cedillo-Ramírez, Lucy E. Correa-Lopez, Jose M. Vela-Ruiz, Leonardo M. Perez-Acuña, Joan A. Loayza-Castro, Camila S. Cabello-Vela, Manuel Huamán-Garcia, Magdiel José Gonzales-Menéndez y Jhony A. De La Cruz-Vargas. Estilos de vida de estudiantes universitarios de ciencias de la salud [Artículo de Revisión]. Rev. Fac. Med. Hum. 2016;16(2):57-65. DOI 10.25176/RFMH.v16.n2.670 


\section{INTRODUCCIÓN}

Los estilos de vida abarcan una serie de dimensiones tales como la actividad física, alimentación, relaciones sociales, aspectos psico-emocionales y espirituales. Estos al mantenerse en un estado óptimo, garantizan que el desarrollo integral del ser humano sea equilibrado'.

Las decisiones que toman las personas sobre sus estilos de vida repercuten tanto a mediano como a largo plazo. Si estas decisiones son inadecuadas en salud, el individuo crearía un riesgo autoimpuesto, que aumentará su predisposicion a enfermar e incluso podría llevarlo a la muerte'. Por el contrario, si las decisiones tomadas son las adecuadas entonces esto garantizaría el mantenimiento de la salud y la promoción de la longevidad ${ }^{2}$. En este sentido, es responsabilidad de cada persona el asegurarse a sí mismo una calidad de vida que garantice el bienestar individual, familiar y social ${ }^{3}$.

Mantener un estilo de vida saludable es especialmente importante entre quienes fomentan y promocionan la salud en la comunidad como los estudiantes universitarios del área de ciencias de la salud. En ellos es frecuente encontrar conductas de riesgo como el sedentarismo, malos hábitos de alimentación e incluso conductas suicidas ${ }^{1}$. Algunos estudios han evidenciado que practicar estilos de vida poco saludable es más común en universitarios que se encuentran en años más avanzados de la carrera ${ }^{1}$; esto podría favorecer al desarrollo de enfermedades metabólicas como el sobrepeso y la obesidad, así como constituir un factor de riesgo para enfermedades cardiovasculares en población joven. Por lo cual, el propósito de este trabajo es presentar una revisión bibliográfica de artículos científicos que aborden el estilo de vida en estudiantes universitarios de ciencias de la salud y su impacto en el bienestar integral.

\section{METODOLOGÍA}

La revisión fue efectuada mediante búsqueda electrónica de artículos relacionados con estilos de vida, en fuentes de indexación ISI, SCIELO, MEDLINE, BVS y PUBMED, considerando con especial énfasis aquéllos realizados en estudiantes universitarios pertenecientes a las escuelas de ciencias de la salud. Los términos de búsqueda fueron: estilo de vida, estudiantes universitarios y ciencias de la salud. Para esta revisión se seleccionaron los artículos publicados con resultados de investigación desde el 2010 a la actualidad y aquellos con aspectos teórico-conceptuales se consi- deró los publicados desde el año 2000. Se analizaron los resultados y se incorporaron los antecedentes en una base de datos para evidenciar similitudes y diferencias que ayuden en la organización del presente artículo.

\section{RESULTADOS Y DISCUSIÓN}

De los 83 artículos revisados, se descartaron 38 por no contar con resultados de investigación en el área de ciencias de la salud, y 12 fueron retirados por ser publicados antes del 2010, quedando solo 33 artículos para esta revisión bibliográfica. De los 33 artículos seleccionados, 18 contaban con resultados de investigación y 15 fueron considerados para aspectos teórico - conceptuales que se relacionan con el propósito del estudio.

\section{Sedentarismo como estilo de vida poco saludable}

El sedentarismo es un problema de salud pública a nivel mundial. Respecto a esto, la OMS menciona que "al menos un $60 \%$ de la población mundial no realiza la actividad física necesaria para obtener beneficios en la salud. Esto se debe a un aumento de los comportamientos sedentarios en el tiempo libre y durante las actividades laborales y domésticas"4. Una persona es sedentaria cuando no realiza una cantidad mínima de movimiento diario (por lo menos entre 25 y 30 minutos), que produzca un gasto energético $>10 \%$ del que ocurre habitualmente al llevar a cabo las actividades cotidianas ${ }^{4}$.

Actualmente, la tecnología es una herramienta que facilita la vida humana. Aunque influye en nuestro estilo de vida trayendo muchos beneficios, también hace que el vivir cotidiano sea más sedentario, favoreciendo el desarrollo de enfermedades relacionadas a estilo de vida hipocinético ${ }^{4}$.

Jiménez y Hernandez (2016) en un estudio sobre "Actividad física y otros hábitos de vida en estudiantes de medicina de la Universidad de Costa Rica" realizado en una muestra de 206 estudiantes, a quienes se les aplicó el "Cuestionario de actividad física cotidiana (PAQ)", evidenció que el $40 \%$ de los participantes no realizaba ningún ejercicio físico, y entre quienes sí lo hacían diariamente, el 68,93\% dedicaba en promedio solamente 7,78 minutos a dicha actividad ${ }^{5}$. Por otro lado, Musaiger recientemente sostuvo que, entre los estudiantes de ciencias de la salud de Bahrain, las mujeres estaban más propensas a mantener comportamientos sedentarios como: ver la televisión, usar el internet, usar el teléfono móvil y dormir ${ }^{6}$. 
Estos resultados remarcan la importancia del enfoque con el que se están formando los nuevos recursos humanos médicos y de salud, no solo en el cuidado de los pacientes a su cargo sino, más importante aún en el autocuidado y la prevención del desarrollo de enfermedades producto de un estilo de vida poco saludable.

\section{La alimentación y su impacto en la salud}

El ritmo de vida actual es cada vez más estresante, es por ello que muchos ahorran tiempo consumiendo lo que les resulte más fácil, sencillo y disponible. Este tipo de conducta ocasiona que las personas estén en riesgo de alimentarse con productos de baja calidad (ricos en triglicéridos, altos en colesterol y azúcar, etc.), ya que el procesamiento y preparación de estos requiere menos tiempo. Esta realidad no es ajena a estudiantes universitarios, mucho menos entre quienes se piensa que son capaces de llevar una forma de vida más saludable, como es el caso de los estudiantes del área de ciencias de la salud. La adquisición y mantenimiento de hábitos alimentarios saludables en los jóvenes puede suponer cambios importantes en el estilo de vida ${ }^{7}$.

Cervera (2013) en un estudio titulado "Hábitos alimentarios y evaluación nutricional en una población universitaria", investigó y aplicó el Índice de Alimentación Saludable (IAS) a 80 estudiantes de la facultad de enfermería de Albacete en Castilla España, evidenciando que la dieta de la población universitaria estudiada es de baja calidad, informando que más del $90 \%$ de la población estudiada necesita hacer "cambios hacia un patrón alimentario más saludable". Además, el estudio mostró que los alimentos que más consumieron los jóvenes fueron los productos cárnicos y lácteos, los cuales tienen elevados niveles de grasa saturada, colesterol y proteína animal; mientras que la ingesta de frutas y verduras fue baja ${ }^{7}$. Rizo-Baeza y col. mostraron que los estudiantes de ciencias de la salud de España manifestaron un desequilibrio en el tipo de grasa ingerida, siendo mayor de lo recomendado el consumo de grasa saturada que el de poliinsaturada ${ }^{8}$.

Así mismo, Soria Trujano (2016) en un estudio realizado en México titulado "Hábitos de alimentación y de sueño en estudiantes de profesiones del área de la salud y de otras áreas" sostiene que de los 100 estudiantes de medicina humana, la mitad de la muestra desayuna, come y cena muy frecuentemente; una menor proporción de estudiantes ingieren, frutas, verduras y leguminosas; y muy pocos estudiantes incluyen pescado en su dieta. Además, casi la mitad de la muestra total come carne roja y pan. Una tercera parte de la muestra estudiada señaló comer golosinas, comida chatarra y reportó que come fuera de casa tortas, quesadillas, tacos, hamburguesas, comida china y/o pollo frito; resultados similares fue observado 100 estudiantes de enfermería?.

Terebessy en un estudio que realizó en 351 estudiantes de medicina de las universidades de Semmelweis, Budapest y Hungría, obtuvo que solo el $15.3 \%$ de la muestra informó tener una dieta saludable. Esto no sorprende, ya que estudios previos han puesto de relieve un cambio en los patrones de dieta saludable; es decir, un aumento en el consumo de alimentos procesados y grasas saturadas y una disminución en la ingesta de alimentos vegetales y ácidos grasos monoinsaturados, aún en países mediterráneos ${ }^{10}$.

Germine El-Kassas y Fouad Ziade en un reciente estudio que realizaron en 369 estudiantes de ciencias de la salud del Norte del Líbano, sostuvieron que los hábitos alimentarios de la muestra reveló la adopción de patrones dietéticos indeseados. Los resultados demostraron que aproximadamente la mitad de los participantes ingieren menos de tres comidas al día, y el $38.5 \%$ de los estudiantes no toma desayuno o lo reemplazan con bocadillos poco saludables $^{11}$. Katarzyna Zaborowicz et al, refiere que estos errores en la alimentación están asociados a un insuficiente conocimiento nutricional, y por tanto, tienen un serio impacto en el estilo de vida de los universitarios $^{12}$.

Las consecuencias negativas de una mala alimentación están claramente demostradas en diferentes estudios. En la Universidad Nacional Mayor de San Marcos donde se evaluó a los internos de la Escuela de Enfermería, se encontró un $12.8 \%$ de sobrepeso entre el alumnado ${ }^{13}$. Mientras que en la facultad de medicina de la Universidad Cayetano Heredia se observó un $26.30 \%$ de sobrepeso y $3.75 \%$ de obesidad en una muestra de 133 universitarios $^{14}$. Además, Dariusz Kałka et al. en una investigación en Polonia sostuvo que una dieta rica en grasa es uno de los factores de riesgo más importantes para desarrollar alguna enfermedad cardiovascular, y tener conocimiento de esto no se relacionaba con el mantenimiento de un estilo de vida saludable entre estudiantes de medicina ${ }^{15}$. 
Diversos autores plantean su preocupación que los estudiantes de ciencias de la salud no apliquen sus conocimientos para el cuidado de su salud requiriéndose un cambio urgente hacia una forma de alimentación más saludable.

\section{Actividad física como práctica necesaria y saludable}

La actividad física no solo abarca el ejercicio planificado, sino también otras actividades que entrañan movimiento corporal y se realizan como parte de los momentos de juego, del trabajo, de formas de transporte activas, de las tareas domésticas y de actividades recreativas ${ }^{16}$.

Es claro el efecto positivo que produce la actividad física en la vida de las personas. Para Cintra y Balboa (2011), la práctica de actividad física debidamente sistematizada (es decir, como ejercicio físico) contribuye a disminuir los efectos del envejecimiento y proporciona un incremento del tono muscular; de esta forma, se genera un aumento en la fuerza. Además, desde el sistema cardiovascular se genera una mejoría en las condiciones hemodinámicas y de la respuesta ventilatoria; como adaptación a la práctica de actividad física se presenta una disminución de la tensión arterial, la hiperlipidemia y la osteoporosis; así como mejoría en la flexibilidad, el equilibrio y la movilidad articular ${ }^{17}$. De manera interesante Aithal y colaboradores, en una investigación que evaluó el impacto que tiene la actividad física sobre el rendimiento académico en estudiantes de medicina de la India, llegaron a la conclusión que la actividad física aumenta la concentración de los estudiantes y mejora el rendimiento académico ${ }^{18}$.

Aunque son evidentes los beneficios de mantener una actividad física diaria, la mayoría de los estudiantes de ciencias de la salud parece no ponerla en práctica; así lo mostró un estudio reciente realizado en Ohio. Los resultados de la investigación revelaron que de los 93 estudiantes de ciencias de la salud que participaron en la investigación, el $44 \%$ practicaban los recomendados 30 minutos de ejercicio 5 días por semana ${ }^{19}$. Así mismo un estudio realizado en México puso de manifiesto que de una muestra de 5745 estudiantes de ciencias de la salud, más de la mitad eran sedentarios ${ }^{20}$.

Por lo antes mencionado, es posible inferir que los sistemas de educación biomédica no son eficientes en la inducción de un estilo de vida saludable entre los estudiantes de ciencias de la salud. Tal fenómeno podría impactar su práctica futura ya que probablemente se convertirán en profesionales obesos, con diversos factores de riesgo y la consecuente falla en la prevención primaria y secundaria de sus propios pacientes ${ }^{20}$.

\section{Tabaquismo, uso de drogas, abuso de alcohol y malos hábitos de sueño}

Múltiples investigaciones evidencian los efectos nocivos del tabaquismo, sobre la salud y aun asi se reportan altos niveles de consumo en el medio universitario. Papathanasiou et al, en un estudio realizado en 1651 estudiantes de ciencias de la salud (690 hombres y 961 mujeres), reportó que la prevalencia de tabaquismo fue del $37,6 \%$ de la muestra de estudio; $y$ el $23,1 \%$ de los consumidores de tabaco fueron considerados fumadores empedernidos ( $\geq 21 \mathrm{cig} /$ día). La prevalencia del tabaquismo no difirió significativamente entre los sexos, pero fumar en exceso fue más prevalente en los hombres ${ }^{21}$.

Además, Guevarra al realizar una investigación sobre el uso del tabaco en 52 estudiantes de ciencias de la salud, reportó que el $79 \%$ de los universitarios afirmaron tener exposición al humo del tabaco en lugares distintos de donde viven ${ }^{22}$; si dentro de estos lugares están los ambientes universitarios, entonces es urgente replantear y aplicar las políticas saludables evitando el consumo de cigarrillos en estos centros de estudios.

Respecto al consumo de drogas ilícitas, la literatura muestra una prevalencia mucho más baja en jóvenes universitarios en general. Helmer et al, en un estudio que busca evaluar el uso de sustancias ilícitas en universitarios de siete países Europeos, reportó que el $8.3 \%$ de los estudiantes consumieron drogas alguna vez en sus vidas ${ }^{23}$. Además, Páez refirió que el consumo de este tipo de sustancias se asocian al ingreso a la universidad y en mayor proporción al primer año de carrera ${ }^{24}$.

En cuanto al abuso de alcohol, según McAlaney esta práctica en adultos jóvenes puede dar lugar a deterioro cognitivo y neurológico; y como consecuencia de estos: bajo rendimiento académico, comportamientos antisociales, prácticas sexuales de riesgo, además de daño físico y psicológico ${ }^{25}$. Sin mencionar la asociación entre alcohol y accidentes automovilísticos en personas jóvenes.

Los hábitos de sueño también son muy investigados como parte de los estilos de vida de los jóvenes 
universitarios. Dormir poco puede constituirse en indicador de un estilo de vida poco saludable y un deficiente estado de salud. Giri y col, en una investigación sobre hábitos y problemas del sueño realizado en 150 estudiantes de medicina de la India, reportó que el $17.3 \%$ de los universitarios tenía niveles anormales de sueño durante el día, generando irritabilidad, deterioro del rendimiento académico y alteración del estilo de vida y de las relaciones interpersonales. Además, la calidad de sueño suele ser mejor en mujeres que en varones ${ }^{26}$. Oleg Pivopsky y colaboradores agrega que de una muestra de 35 residentes de medicina, el 15\% de los participantes sufrieron accidentes automovilísticos, y el $49 \%$ del total de la muestra refirió haber tenido error de juicio durante la práctica médica ${ }^{27}$.

Las publicaciones revisadas muestran que la práctica de hábitos nocivos para la salud y bienestar humano afecta a la población universitaria y que estos indices pueden ir en aumento si no se enfatiza la identificación temprana de estas conductas de riesgo en jóvenes universitarios. Por ello, es urgente la promoción de estilos de vida saludable en los ambientes universitarios.

\section{Bienestar mental como determinante para un estilo de vida saludable}

La salud mental es importante en toda persona para alcanzar el equilibrio en la vida. La alimentación y la actividad física contribuyen en el logro del bienestar psicológico y social; pero también hay otros factores como la personalidad, las emociones y su forma de relacionarse socialmente, por lo cual esta dimensión se centra en el desarrollo personal, en la forma en que las personas afrontan los retos vitales y sus esfuerzos por conseguir sus metas, así también en las valoraciones que hacen de las circunstancias $y$ de su funcionamiento dentro de la sociedad ${ }^{28}$.

Mantener niveles de responsabilidad y productividad altos, permite conservar niveles psicosociales equilibrados, y evita que se desencadenen enfermedades de tipo psicosocial, como la depresión o la ansiedad ${ }^{3,29}$. Un aspecto esencial esta relacionado al bienestar espiritual de las personas, el cual les genera esperanza para confrontar situaciones que conllevan a la trascendencia y la incertidumbre, promoviendo la fe y practicando la religiosidad y la fortaleza interior, de manera que les brinde el equilibrio emocional idóneo ${ }^{3}$. Respecto a esto, Laguado
Jaimes (2014) en un estudio realizado en Colombia en estudiantes de la escuela de enfermería, demostró que los universitarios desarrollan mayor autodeterminación y autorregulación cuando creen en un ser superior, tienen propósito en la vida y están en paz consigo mismos ${ }^{30}$.

Definitivamente, el bienestar mental produce estilos de vida positivos que se sustentan en la humanidad, confianza, seguridad y sensación de control sobre su propio destino, y le da a la vida opción, sentido, respeto y legitimidad ${ }^{31}$. Sostener una salud mental óptima es especialmente importante en poblaciones universitarias, ya que contribuye a un mejor desempeño en el campo académico y aumenta las capacidades de afrontamiento ante situaciones de estrés y ansiedad que vienen junto con la etapa de preparación académica. Ybarra Sargarduy (2011) en un estudio titulado "Salud Mental y Estilos de Vida en Estudiantes Universitarios" sostiene que uno de cada cinco alumnos universitarios participantes, presenta alteraciones de salud mental; el consumo de tabaco y/o drogas, aparece como un factor de riesgo en las alteraciones de la salud mental, mientras que la actividad física, destaca como un factor de protección ${ }^{32}$.

Jessica Slonim y colaboradores (2015), sostuvo que el cuidado de uno mismo en dimensiones como: salud, actividad física, nutrición, espiritualidad, entre otros; potencian el bienestar mental y disminuyen la angustia ${ }^{33}$.

La tabla 1, muestra los 19 artículos con resultados de investigación en estilos de vida; destacándose la procedencia, las fortalezas y las debilidades de cada uno. En la Tabla 2, se detallan los estudios que utilizaron algún instrumento para medir estilos de vida o alguna de sus dimensiones en estudiantes de ciencias de la salud. Finalmente en la Tabla 3 se hace una comparación de los resultados obtenidos a partir de las fuentes consultadas para esta revisión, pero diferenciándolos según género.

La presente revisión bibliográfica fue elaborada por nuestro grupo de investigación ESVIBUR que pertenece al Instituto de Investigación en Ciencias Biomédicas (INICIB) de la Universidad Ricardo Palma, y forma parte de la linea de investigacion: "Estilo de Vida en Estudiantes Universitarios" del área de ciencias de la salud. 
Tabla 1. Fortalezas y debilidades de las 18 fuentes científicas seleccionados para el artículo de revisión.

\section{Título}

\section{Procedencia}

Debilidades

Actividad física y otros hábitos de vida en estudiantes de medicina de la Universidad de Costa Rica ${ }^{5}$.

Costa Rica 2016

Bahréin 2015

Dietary habits and sedentary behaviors among health science university
Fortalezas

Se utilizó un instrumento adaptado por uno de los autores.
No realiza un análisis detallado sobre el sedentarismo. students in Bahrain ${ }^{6}$. nutricional en una población universitaria ${ }^{7}$.
Hábitos alimentarios y evaluación

tudiantes de ciencias de la salud ${ }^{8}$.

Hábitos de alimentación y de sueño en

la salud y de otras áreas. Análisis entre estudiantes de profesiones del área de géneros 9 .

México (2016)

Medical students' health behavior and self-reported mental health status by their country of origin: a cross-sectional study ${ }^{10}$.

Exploration of the Dietary and Lifestyle Behaviors and Weight Status and Their Self-Perceptions among Health Sciences University Students in North Lebanon" ${ }^{11}$.

(2016)

España

(2013)

\section{España Demuestra que los conocimientos 2014

cisiones.

Relacionar los patrones dietarios con el sedentarismo.

Caracterización de hábitos alimentarios y calidad de dieta.
La cantidad de varones fue más pequeña que la de mujeres.

La población estudiada abarca solo e primer curso de enfermería.
Evaluation of selected dietary behaviors of students according to gender and nutritional knowledge ${ }^{12}$. Silesian students of the Faculty of Medicine: Knowledge and distribution ${ }^{15}$.

The Impact of Physical Activities on the Academic Performance of Medical Students ${ }^{18}$.

Physical health, lifestyle beliefs and behaviors, and mental health of entering graduate health professional students: Evidence to support screening and early intervention ${ }^{19}$.

Anthropometric traits, blood pressure, and dietary and physical exercise habits in health sciences students; The Obesity Observatory Project ${ }^{20}$.

\section{Smoking and Physical Activity Interre-} lations in Health Science Students. Is Smoking Associated with Physical Inactivity in Young Adults? ${ }^{21}$.

Tobacco Use Among Fourth Year Bachelor of Science in Public Health (BSPH) Students of the College of Public Health: University of the Philippines Manila, Academic Year 2012-201322.

\begin{tabular}{|c|c|c|c|}
\hline $\begin{array}{l}\text { Study of Sleep Habits and Sleep Pro- } \\
\text { blems Among Medical Students of Pra- } \\
\text { vara Institute of Medical Sciences Loni, } \\
\text { Western Maharashtra, India }{ }^{26} \text {. }\end{array}$ & $\begin{array}{l}\text { India } \\
2013\end{array}$ & $\begin{array}{l}\text { Estudia la relación entre malos hábitos } \\
\text { de sueño y desórdenes psiquiátricos. }\end{array}$ & $\begin{array}{l}\text { Diseño transversal, los resultados po- } \\
\text { drían no ser representativos de la po- } \\
\text { blación en general. }\end{array}$ \\
\hline $\begin{array}{l}\text { The impact of sleep deprivation on slee- } \\
\text { piness, risk Factors and Professional Per- } \\
\text { formance in medical residents }{ }^{27} \text {. }\end{array}$ & $\begin{array}{l}\text { Israel } \\
2013\end{array}$ & $\begin{array}{l}\text { Asocia la falta de sueño con los desór- } \\
\text { denes metabólicos y bajo rendimiento } \\
\text { profesional. }\end{array}$ & $\begin{array}{l}\text { Estudio piloto, de diseño transversa } \\
\text { con muestra pequeña. }\end{array}$ \\
\hline $\begin{array}{l}\text { Estilos de vida saludable en estudian- } \\
\text { tes de enfermería de la universidad } \\
\text { cooperativa de Colombia }{ }^{30} \text {. }\end{array}$ & $\begin{array}{l}\text { Colombia } \\
2014\end{array}$ & $\begin{array}{l}\text { Aporta que los hábitos saludables no } \\
\text { son rutinarios. }\end{array}$ & $\begin{array}{l}\text { Solo participan } 154 \text { estudiantes. Sexo } \\
\text { predominante: femenino. }\end{array}$ \\
\hline $\begin{array}{l}\text { The relationships among self-care, dis- } \\
\text { positional mindfulness, and psycholo- } \\
\text { gical distress in medical students }{ }^{33} \text {. }\end{array}$ & $\begin{array}{l}\text { Estados Unidos (Kentucky) } \\
2015\end{array}$ & $\begin{array}{l}\text { Estudia la relación entre el auto cuida- } \\
\text { do y los niveles de angustia. }\end{array}$ & $\begin{array}{l}\text { La cantidad de varones de la muestra es } \\
\text { mucho menor a la cantidad de mujeres. }\end{array}$ \\
\hline
\end{tabular}

studia la relación entre la calidad de die-

ta y los conocimientos sobre nutrición.
Cardiovascular risk factors among lower
Estados Unidos 2016

México 2013

\section{Grecia} 2012

Filipinas

ungría

2016

Líbano

Polonia

2016

olonia

2016

India

016

16

Se evaluó los hábitos alimentarios, comparándolos según área profesional y según género.

Existe fuerte asociación entre el lugar de origen y la salud mental.

Identifica factores relacionados a una mala dieta que influyen en el desarrollo de obesidad.

El diseño transversal no permite revelar tendencias de cambio en los hábitos alimentarios.
No se evalúa la relación entre imagen corporal y la supresión de comidas al día. os estudios de diseño transversal no permite sacar conclusiones de la relaciones de causa - efecto. 
Tabla 2. Estudios en los que se utilizaron instrumentos para medir estilos de vida o alguna de sus dimensiones en estudiantes de ciencias de la salud.

México Escala tipo likert de 65 items $^{9}$
The Healthy Lifestyle Beliefs Scale ${ }^{19}$
100 alumnos de cada una de las carreras: medicina, enfermería, arquitectura y comunicación.
Diseñado en el 2016. Evalúa las dimensiones de actividad física, automedicación autocuidado, actividad sexual, hábitos de sueño y hábitos alimentarios. No incluye una dimensión específica para la evaluación del bienestar mental.

535 alumnos de la Universidad Autónoma de Tamaulipas.
Confiabilidad de 0.80 . Se utilizó la adaptación antigua del cuestionario.

\section{Colombia HPLP de Nola Pender ${ }^{30}$ \\ Escala de likert de 4 puntos. Fiabilidad alfa de 0.93 Alfa de Cronbach de 0.70 a 0.87 .} procedentes de 7 universidades de Estados Unidos.

The Healthy Lifestyle Beliefs Scale: 16 ítems, likert 5 y alfa de cronbach de 0.84 .

\section{The Healthy Lifestyle Behaviors Scale ${ }^{19}$ \\ 93 estudiantes de ciencias de la salud de procedentes de 7 universidades de Estados Unidos.}

The Healthy Lifestyle Behaviors Scale: 15 ítems, likert 5 y alfa de cronbach de 0.75 .

52 ítems con 6 subdimensiones: autocuidado, nutrición, actividad física, crecimiento espiritual, relaciones interpersonales $y$ manejo del estrés. Likert 4. Coeficiente alfa: 0.94 . dad de Australia.
(HPLP II) $)^{33}$

Hungría

Simple Lifestyle Indicator Questionnaire ${ }^{10}$

estudiantes de
369 estudiantes de ciencias de la salud de cuatro universidades del norte del Líbano.

456 estudiantes de diferentes facultades (se incluye ciencias de la salud)

Adaptado a la realidad de Hungría y publicado en el 2016. Likert 5.

Cuestionario desarrollado por el autor y publicado durante el 2016.

Incluye dimensiones como: nutrición y cambios en la dieta, actividad física y mediciones antropométricas.

Preguntas cerradas para evaluar los comportamientos nutricionales. 26 preguntas para responder verdadero o falso en cuanto a la nutrición.

2000 estudiantes de ciencias de la salud de Grecia.

Alta fiabilidad y adecuada validez para poblaciones de adultos jóvenes.
Bahréin Cuestionario autoadministrado sobre hábitos alimentarios y sedentarismo ${ }^{6}$
642 estudiantes de ciencias de la salud.

Coeficiente de correlación de pearson: 0.87
India Cuestionario sobre actividad física y su impacto en el rendimiento académico ${ }^{26}$
210 estudiantes de medicina de Malasia.

Cuestionario sobre hábitos de tabaquis-

Costa Rica mo e ingesta de alcohol; frecuencia de consumo de alimentos (FFQ), y de actividad física cotidiana $(\mathrm{PAQ})^{5}$
206 estudiantes de medicina de la Universidad de Costa Rica.

184 estudiantes de ciencias de la salud (enfermería y nutrición).
Modificado de acuerdo a los objetivos del estudio. Constituido por 11 preguntas cerradas. Incluye ítems para evaluar el rendimiento académico.

El cuestionario fue desarrollado a partir de versiones previamente validadas. Publicado en el 2016.

Dos partes: La primera contiene ítems para medir estilos de vida y registrar medidas antropométricas. La segunda tiene un recordatorio de la ingesta de alimentos en 24 horas. 
Tabla 3. Comparación de resultados, según género, obtenidos a partir de las fuentes consultadas para la presente revisión.

\begin{tabular}{|c|c|c|c|c|c|c|}
\hline \multirow[t]{2}{*}{ Titulo } & \multirow[t]{2}{*}{$\begin{array}{l}\text { Población según } \\
\text { sexo }\end{array}$} & Actividad física (\%) & $\begin{array}{l}\text { Alimentación } \\
\text { Saludable (\%) }\end{array}$ & \multicolumn{2}{|c|}{$\begin{array}{c}\text { Hábitos } \\
\text { Nocivos (\%) }\end{array}$} & Salud mental (\%) \\
\hline & & $\mathbf{M}$ & $\mathbf{M}$ & $\mathbf{F}$ & $\mathbf{M}$ & $\mathbf{M}$ \\
\hline
\end{tabular}

Dietary habits and sedentary behaviors among health science university stu552 Mujeres;
90 Varones $16 \quad$ AF diaria: Desayunan diariadents in Bahrain ${ }^{6}$.

16

41.6

44.2 44.4

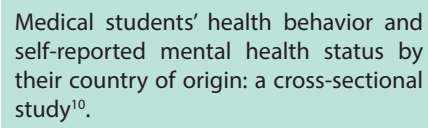

\section{Mujeres; \\ 267 Varones}

$75 \%$ de la muestra total tiene una rutina vigorosa de ejercicios (Varones con más frecuencia que mujeres).
15.3\% de la muestra Alcohol: $13.8 \%$ de tiene una dieta salu- la muestra total dable (Mujeres con (Mujeres en igual los varones). mayorfrecuencia que proporción que
El bienestar mental de las mujeres es semejante al de los varones en esta muestra.
Evaluation of selected dietary behaviors of students according to gender and nutritional knowledge $\mathrm{e}^{12}$.
277 Mujeres;
179 Varones
34.7

25.1
Physical health, lifestyle beliefs and behaviors, and mental health of entering graduate health professional students: Evidence to support screening and early intervention ${ }^{19}$.
Smoking and Physical Activity Interrelations in Health Science Students. Is Smoking Associated with Physical Inactivity in Young Adults? ${ }^{21}$

\begin{tabular}{ccc} 
& \multicolumn{2}{c}{ AF vigorosa: } \\
961 Mujeres; & 10.9 & 18.7 \\
690 Varones & AF moderada: \\
& 39.6 & 41.6
\end{tabular}

Hábito de fumar:

$36.1 \quad 39.6$

Tobacco Use Among Fourth Year Bachelor of Science in Public Health (BSPH)

Students of the College of Public Health University of the Philippines Manila, Aca-

Study of Sleep Habits and Sleep Problems Among Medical Students of Pravara Institute of Medical Sciences Loni, Western Maharashtra, India ${ }^{25}$

\section{Mujeres;}

98 Varones

Fuente: ESVIBUR - INICIB

\section{CONCLUSIÓN}

Las publicaciones consultadas demuestran que el estilo de vida en estudiantes universitarios, especialmente del área de ciencias de la salud, tiende a ser poco saludable. Esta realidad obliga a tomar medidas que promuevan un cambio hacia estilos de vida saludables; para esto, es necesaria la evaluación del estilo de vida mediante un instrumento de medición adecuado. Los estudios citados aplicaron diferentes escalas y cuestionarios, pero pocos han sido adaptados a la realidad peruana. Por ello es necesario realizar futuras investigaciones que aborden esta problemática.

Financiamiento: Autofinanciado.

Conflicto de interés: Los autores declaran no tener conflictos de interés en la publicación de este artículo.

Recibido: 15 de Junio de 2016

Aprobado: 22 de Julio de 2016 


\section{REFERENCIAS BIBLIOGRÁFICAS}

1. Bastías EM, Stiepovich J. Una revisión de estilos de vida de estudiantes universitarios iberoamericanos. Ciencia y enfermería. 2014; XX(2):93-101.

2. Roca M. Desarrollo autónomo del individuo y la responsabilidad en la prevención: problema de salud. Cultura, Educación y Sociedad. 2010; 1(1):133-145.

3. Sala J. Estilos de vida saludable: Un derecho fundamental en la vida del ser humano. Revista Latinoamericana de Derechos Humanos. 2015; 26(2):37-51.

4. Organización Mundial de la Salud. Estrategia mundial sobre el régimen alimentario, actividad física y salud. Informe sobre la salud en el mundo 2002: reducir los riesgos y promover una vida sana. Ginebra: OMS; 2002. Serie de Informes Técnicos: 180 .

5. Jiménez S, Hernández Elizondo. Actividad física y otros hábitos de vida en estudiantes de medicina de la Universidad de Costa Rica. Revista de Ciencias del Ejercicio y la Salud. 2016; 14(1):1-14.

6. Musaiger AO, Awadhalla M, Al-Mannai M, AlSawad M, G.V. Asokan. Dietary habits and sedentary behaviors among health science university students in Bahrain. Adolesc Med Health. 2015; aop.

7. Cervera F, Serrano B, Vico C, Milla M, García MJ. Hábitos alimentarios y evaluación nutricional en una población universitaria. Nutr Hosp. 2013;28(2):438-446

8. Rizo-Baeza M.M, González-Brauer N,G, Cortés E. Calidad de dieta y estilos de vida en estudiantes de ciencias de la salud. Nutr Hosp. 2014;29(1):153-157.

9. Soria R, Ávila E, Feliciano A. Hábitos de alimentación y de sueño en estudiantes de profesiones del área de la salud y de otras áreas. Análisis entre género. [Revista en línea]. 2016 [Consultado 4 octubre 2016]; 19(1). Disponible en: http://www.revistas.unam.mx/index.php/repi.

10. Terebessy A, Czeglédi E, Balla BC, Horváth F, Balázs P. Medical students' health behaviour and self-reported mental health status by their country of origin: a cross-sectional study. BMC Psychiatry. 2016; 28(16):171.

11. El-Kassas G, Ziade F. Exploration of the Dietary and Lifestyle Behaviors and Weight Status and Their Self-Perceptions among Health Sciences University Students in North Lebanon. Biomed Res Int. 2016;2016:9762396.

12. Zaborowicz K, Czarnocińska J, Galiński G, Kaźmierczak P, Górska K, Durczewski P. Evaluation of selected dietary behaviours of students according to gender and nutritional knowledge. Rocz Panstw Zakl Hig 2016;67(1):45-50.

13. Monge J. Hábitos alimentarios y su relación con el índice de masa corporal de los internos de enfermería de la U.N.M.S.M. [Tesis]. Lima: Universidad Nacional Mayor de San Marcos. Facultad de Medicina Humana; 2007

14. Llanos F, Najar N, Mayca J, Rosas A. Prevalencia de obesidad e hipercolesterolemia en la Facultad de Medicina de la Universidad Peruana Cayetano Heredia - 1998. Rev Med Hered. 2001;12(3):78-84.

15. Kalka, D., et al. Cardiovascular Risk Factors Among Lower Silesian Students of the Faculty of Medicine: Knowledge and Distribution. Advances in clinical and experimental medicine: official organ Wroclaw Medical University. 2016;25(2): 341.

16. Organización Mundial de la Salud. Recomendaciones mundiales sobre actividad física para la salud. Ginebra: OMS; 2010. Serie de Informes Técnicos: 255.

17. Cintra O, Balboa Y. La actividad física: un aporte para la salud. [Revista en línea]. 2011. [Consultado 4 octubre 2016]; 16 (159). Disponible en:h- ttp://www.efdeportes.com/efd159/la-actividad-fisica-para-la-salud.htm.

18. Aithal A. P. The impact of physical activities on the academic performance of medical students. Online Journal of Health and Allied Sciences. 2016; 15(2).

19. Mazurek B, et al. Physical health, lifestyle beliefs and behaviors, and mental health of entering graduate health professional students: Evidence to support screening and early intervention. Journal of the American Association of Nurse Practitioners. 2016; 28(4):204-211.

20. Gutiérrez-Salmeán G, Meaney A, Ocharán ME, Araujo JM, Ramírez-Sánchez I, Olivares-Corichi IM, et al. Anthropometric traits, blood pressure, and dietary and physical exercise habits in health sciences students; The Obesity Observatory Project. Nutr Hops. 2013; 28(1):194-201.

21. Papathanasiou G, Papandreou M, Galanos A, Kortianou E, Tsepis E, Kalfakakou V, Evangelou A. Smoking and physical activity interrelations in health science students. Is smoking associated with physical inactivity in young adults? Hellenic J Cardiol. 2012;53(1):17-25.

22. Guevarra JP, Cordova RQ Jr, Mercado CE, Asaad AS. Tobacco use among fourth year Bachelor of Science in Public Health (BSPH) students of the College of Public Health: University of the Philippines Manila, academic year 2012-2013. J Community Health. 2014 Oct;39(5):886-93.

23. Helmer SM, Mikolajczyk RT, McAlaney J, Vriesacker B, Van Hal G, Akvardar $Y$, et al. Illicit substance use among university students from seven European countries: a comparison of personal and perceived peer use and attitudes towards illicit substance use. Prev Med. 2014;67:204-9.

24. Páez M. Universidades saludables: los jóvenes y la salud. Archivos de Medicina (Col) [en linea]. 2012. [Fecha de consulta: 5 de octubre de 2016] Disponible en: http://www.redalyc.org/articulo.oa?id=273825390007.

25. McAlaney J, Helmer SM, Stock C, Vriesacker B, Van Hal G, Dempsey RC, et al. Personal and Perceived Peer Use of and Attitudes Toward Alcohol Among University and College Students in Seven EU Countries: Project SNIPE. J Stud Alcohol Drugs. 2015;76(3):430-8.

26. Giri P, Baviskar M, Phalke D. Study of sleep habits and sleep problems among medical students of pravara institute of medical sciences loni, Western maharashtra, India. Ann Med Health Sci Res. 2013;3(1):51-4

27. Pikovsky O, Oron M, Shiyovich A, Perry ZH, Nesher L. The impact of sleep deprivation on sleepiness, risk factors and professional performance in medical residents. Isr Med Assoc J. 2013;15(12):739-44.

28. Zubieta E, Muratori M, Fernandez O. Bienestar subjetivo y psicosocial: explorando diferencias de género. Salud \& sociedad. 2012; 3(1):66-76

29. Otero C, Gálvez M. Plan de retorno al trabajo tras baja laboral por motivos de salud mental. Med Segur Trab. 2014; 60(235): 392-405

30. Laguado Jaimes E, Gómez Díaz MP. Estilos de vida saludable en estudiantes de Enfermería en la Universidad Cooperativa de Colombia. Hacia promoc. salud. $2014 ; 19(1): 68-83$

31. Calderón Jorge. Salud mental, sociedad y cotidianidad. 2001; 1-25.

32. Sagarduy J, et al. Salud mental y estilos de vida en estudiantes universitarios. Tópicos en psicología. 2011; 75-84.

33. Slonim J, Kienhuis M, Di Benedetto $M$, Reece J. The relationships among self-care, dispositional mindfulness, and psychological distress in medical students. Med Educ Online. 2015, 20: 27924. 